\title{
Retinal nerve fibre layer thickness values and their associations with ocular and systemic parameters in Black South Africans.
}

\author{
Khathutshelo P. Mashige, MOptom, BOptom, ${ }^{1}$ \\ Olalekan A. Oduntan,Optom ${ }^{2}$
}

1. Discipline of Optometry, School of Health Sciences,

2. University of KwaZulu-Natal, Private Bag X54001, Durban, 4000 South Africa

\begin{abstract}
Purpose: To measure the retinal nerve fibre layer (RNFL) thickness values and investigate their associations with other parameters in healthy eyes of Black South Africans.

Methods: 600 participants with healthy eyes, of whom 305 (50.83\%) were males and $295(49.17 \%)$ were females, with a mean age of $28.15 \pm 13.09$ years, underwent a detailed ophthalmic examination. RNFL thickness was measured by iVue SD-OCT.

Results: The mean global RNFL thickness was $110.01 \pm 7.39 \mu \mathrm{m}$. The RNFL was thickest inferiorly $(135.06 \pm 9.66 \mu \mathrm{m})$ and superiorly $(131.72 \pm 10.46 \mu \mathrm{m})$, thinner nasally $(87.24 \pm 13.22 \mu \mathrm{m})$, and thinnest temporally $(73.63 \pm 15.66 \mu \mathrm{m})$. Multivariate analysis showed that thicker mean global RNFL thickness was significantly associated with younger age, shorter axial length (AL) and hyperopia $(\mathrm{p}<0.001)$. Mean RNFL thickness decreased by approximately $0.11 \mu \mathrm{m}$ per year of aging life, and by $1.02 \mu \mathrm{m}$ for each 1-mm of axial elongation. There was a $0.62 \mu \mathrm{m}$ RNFL thickness increase for every dioptre change in spherical power towards more hyperopia.

Conclusion: Mean RNFL thickness values and their associations established in this population may be of clinical value when assessing factors that influence this parameter and diagnosing diseases affecting it.

Keywords: Retinal nerve fibre layer, optical coherence tomography, refractive error, axial length, glaucoma.

DOI: http://dx.doi.org/10.4314/ahs.v16i4.39

Cite as: MashigeKP, Oduntan $O A$. Retinal nerve fibre layer thickness values and their associations with ocular and systemic parameters in Black. South Africans. Afri Health Sci. 2016;16(4): 1188-1194. http:/ / dx.doi.org/10.4314/abs.v16i4.39
\end{abstract}

\section{Introduction}

Glaucoma is the second leading cause of global blindness, its prevalence varying among different racial groups. ${ }^{1}$ Primary open angle glaucoma has been reported to be more prevalent amongst Black African people, while angle closure glaucoma is commonest amongst Asians. ${ }^{1}$ This condition is characterised by progressive optic neuropathy, accelerated damage of the retinal ganglion cells and axons, and thinning of the retinal nerve fibre layer (RNFL) and neuroretinal rim. ${ }^{2}$ Recent evidence suggests that loss

\section{Corresponding author: \\ Khathutshelo P. Mashige, \\ Discipline of Optometry, \\ School of Health Sciences, \\ University of KwaZulu-Natal, \\ Private Bag X54001, Durban, \\ 4000 South Africa, \\ E-mail: mashigek@ukzn.ac.za}

of ganglion cells and anomalies of the RNFL occur before functional deficits become manifest in the visual fields. ${ }^{3}$ Objective, quantitative and sensitive assessment methods of the RNFL thickness are therefore essential to diagnose and monitor progression of optic nerve diseases, such as glaucomatous optic neuropathy and other neurological causes. ${ }^{4}$

Optical coherence tomography (OCT) is a non-invasive, non-contact imaging technique that provides real time, objective and in-vivo cross-sectional images of the retina and its sub-structures, being useful to diagnose and manage a variety of retinal diseases and glaucoma. ${ }^{5}$ OCT works on the principle of interferometry, which uses reflected light to determine the interface between different ocular tissues and create a cross-sectional image (tomogram) of the tissue of interest. ${ }^{5}$ The RNFL fibres appear as a highly reflective layer due to their unique perpendicular arrangement in relation to the direction of the OCT light beam, which allows its borders to be automatically detected and its thickness measured using computer al- 
gorithms. ${ }^{5}$ The recently developed spectral domain (SDOCT) technology ${ }^{5}$ has a five times higher image resolution and 60 times faster imaging speed compared with the conventional time domain (TD-OCT) devices, resulting in improved diagnostic accuracy in detecting early glaucoma. ${ }^{6,7,8}$

Normative databases of RNFL thickness values from age-matched normals are used to interpret thickness measurements generated by SD-OCT machines. These values vary and cannot be used interchangeably between different SD-OCT machines. ${ }^{9}$ In addition, studies ${ }^{10,11}$ have shown that RNFL varies with age, gender, race and ethnicity, refractive error, axial length and disc area, which indicates the need for the normal RNFL thickness values to be determined for each ethnic group. Furthermore, early diagnosis of glaucoma requires accurate and reliable measurement of RNFL thickness, as well as adequate knowledge of the normal values of the RNFL thickness and optic disc configuration in normal subjects. This study was therefore conducted to establish normal values of the RNFL using the iVue SD-OCT, and to investigate their associations with systemic and ocular parameters. The systemic variables include age, gender, height $(\mathrm{H})$, weight (W), body mass index (BMI), systolic and diastolic pressure, while the ocular variables include spherical equivalent refractive error (SE), axial length (AL), anterior chamber depth (ACD), crystalline lens thickness (LT), corneal diameter (CD), anterior corneal curvature (ACC), central corneal thickness (CCT) and intraocular pressure (IOP) in order to optimise the predictive power of this instrument for ocular diseases management.

\section{Subjects and methods}

This cross-sectional study was conducted at the Eye Clinic of the University of KwaZulu-Natal, Durban, South Africa. After obtaining ethical approval from the University of KwaZulu-Natal's Biomedical Ethics and Research Ethics Committee, 600 subjects with no ocular problems, other than refractive error, were enrolled for the study. The subjects were selected through stratified random cluster sampling in six districts of Durban, a major City in the Kwa-Zulu Natal Province of South Africa. To obtain a $95 \%$ confidence level at a standard deviation of 5 , the minimum sample size needed for the study sample was 384 subjects. This number was obtained by using the formula: $\mathrm{n}=(\mathrm{z} \times \mathrm{s})^{2} / \mathrm{e}^{2}$, where $\mathrm{n}=$ sample size, $\mathrm{z}=$
$1.96(95 \%$ confidence interval $), \mathrm{s}=$ standard deviation of 5 , e $=$ deviation of $0.5 .{ }^{12}$ Two field workers approached the subjects in their houses and informed them about the purpose of the study. Those who agreed to participate in the study were transported by one field worker to the examination site, while the other fieldworker moved to the next selected site.

The study protocol adhered to the tenets of the Declaration of Helsinki, and all participants gave accent or consent after the nature of the study had been explained to them. All subjects underwent a complete ophthalmic examination, including a review of medical history, refraction, assessment of ocular motility and alignment, assessment of IOP, slit-lamp biomicroscopic examination, standard automated perimetry, and a fundus examination. Subjects with best-corrected visual acuity of worse than $20 / 20$, IOP $>21 \mathrm{mmHg}$, visual field loss, history of ocular diseases or trauma, intracranial disease and diabetes mellitus, intraocular surgery or any kind of laser therapy, amblyopia or strabismus, abnormalities of the disc or RNFL were excluded. The other exclusion criteria were: media opacity, history of glaucoma, or any other hereditary eye disease, history of neurological, metabolic, vascular disorders, or other systemic disease possibly affecting the eye.

Body height and weight were recorded, and blood pressure (BP) was measured in $\mathrm{mmHg}$. Height was measured in centimetres $(\mathrm{cm})$ with a tape measure and weight was measured in kilograms (kg) with a digital Adam equipment scale, while BP was assessed with an automated Rossmax International MediPro 100F BP device. These measurements were taken three times and averaged. BMI was established by dividing the weight in kilograms $(\mathrm{kg})$ by height in metres squared $\left(\mathrm{m}^{2}\right)$ or $\mathrm{BMI}=\mathrm{kg} / \mathrm{m}^{2}$. Refractive errors were determined by both autorefraction (with theNidek AR-310A) and subjective refraction. Subjective refraction results were used to determine the relevant spherical equivalents, which was defined as the spherical power plus half of the minus cylindrical power (sphere $+1 / 2$ cylinder). IOP measurements were taken using the Nidek NT530P Tonopachy. AL, ACD and LT were measured using the Nidek US-500 Echoscan, while $\mathrm{CD}$ and ACC were measured with the Oculus Keratograph 4 (OCULUS Optikgeräte $\mathrm{GmbH}$ ). The iVue-100 SD-OCT (Optovue Inc. Fremont, CA, USA) device was 
used to measure CCT and RNFL thickness. The iVue-100 SD-OCT scans faster (26,000 A-scans per second) and provides higher resolution cross-sectional images $(5 \mu \mathrm{m})$ than the TD-OCT. ${ }^{5,67,8}$ RNFL measurements were performed using the optic nerve head (ONH) iVue-100 (Optovue, Inc.) protocol, which consists of 12 radial scans $3.4 \mathrm{~mm}$ in length and 13 concentric ring scans ranging from 1.3 to $4.9 \mathrm{~mm}$. RNFL thickness was measured along a circle of $3.45 \mathrm{~mm}$ diameter centred on the optic disc, with the outputs from the measurements being temporal, superior, nasal, and inferior average. All measurements were taken with the eyes in their natural undilated state, and three consecutive scans were performed successively, with their average being computed and used in the analyses. All scans were performed by the same examiner and the scans were accepted only if they were free of artefacts and had signal strengths of at least 40 (as suggested by the manufacturer).

All statistics were done using the Stata: Data analysis and statistical software (Version 11.0, Texas, USA). Analysis of variance (ANOVA) between groups was performed in different quadrants. Pearson correlation coefficients were determined to examine the correlation of RNFL values between the two eyes. The Kolmogorov-Smirnov test was used to evaluate the normality of the distributions of the RNFL thickness data. Descriptive statistics such as ranges, means, standard deviations and confidence intervals were used to determine the main outcome parameter. Univariate analyses were performed to determine the associations between RNFL thickness and ocular and systemic parameters. Multivariate regression analysis was performed with RNFL thickness as the dependent parameter and all systemic and ocular parameters as independent variables, which were associated significantly with RNFL thickness in the univariate analyses. All p-values were two-sided, and a probability $(\mathrm{p})$ value $<0.05$ was considered to be statistically significant.

\section{Results}

Participants included $305(50.83 \%)$ males and 295 (49.17\%) females, with the ages ranging from 10 to 66 years. Regarding the RNFL thickness of the right and left eyes, there was a strong correlation, $(r=0.934, p<0.01)$ the results of the right eyes are therefore presented here. The mean RNFL thickness of all subjects was $110.01 \mu \mathrm{m}$ \pm 7.39 (95\% CI: 109.06-111.95). The mean RNFL thickness was highest in the inferior quadrant, $135.06 \pm 9.66$ (95\% CI: 134.63-136.22); followed by superior, 131.96 \pm 10.46 (95\% CI: 129.10-132.81); nasal, $87.24 \pm 13.22$ (95\% CI: 86.98-89.41); and temporal quadrants, $73.63 \pm$ 15.66 (95\% CI: 71.02-75.05). RNFL thickness values in all the four quadrants differed at the $\mathrm{p}<0.05$ levels.

A decrease in RNFL thickness was significantly associated with increasing age, with Figure 1 showing a significant negative linear relationship between age and global thickness ( $p<0.001)$. Decreasing RNFL thickness was also significantly $(\mathrm{p}<0.001)$ associated with increasing myopia (Figure 2). In the univariate analysis, thicker RNFL thickness was associated with systemic variables such as shorter individuals ( $p<0.001)$, and with ocular parameters such as shorter AL $(\mathrm{p}<0.001)$, thinner CCT $(\mathrm{p}=$ $0.02)$, and lower intraocular pressure $(\mathrm{p}=0.004)$. Other associations from the univariate analysis are shown in Table 1. 
Table 1: Univariate analysis of associations between mean global retinal nerve fibre layer thickness and systemic and ocular parameters.

\begin{tabular}{|c|c|c|c|c|}
\hline Parameter & $\begin{array}{l}p- \\
\text { value }\end{array}$ & $\begin{array}{l}\text { Regression } \\
\text { coefficient B } \\
\text { (or Mean } \\
\text { difference) } \\
\end{array}$ & $\begin{array}{l}\text { Standard } \\
\text { coefficient } \\
\text { beta }\end{array}$ & $\begin{array}{l}5 \% \text { CI of B or } \\
\text { Iean difference }\end{array}$ \\
\hline Age (years) & $<0.001$ & -0.12 & -0.09 & $-0.18,-0.09$ \\
\hline Gender & 0.66 & & & \\
\hline Body height $(\mathrm{cm})$ & $<0.001$ & -0.15 & -0.06 & $-0.14,-0.03$ \\
\hline Body weight (kg) & 0.87 & & & \\
\hline $\operatorname{BMI}\left(\mathrm{kg} / \mathrm{m}^{2}\right)$ & 0.04 & 0.11 & 0.04 & $0.02,0.22$ \\
\hline Systolic blood pressure $(\mathrm{mmHg})$ & 0.42 & & & \\
\hline Diastolic blood pressure $(\mathrm{mmHg})$ & 0.77 & & & \\
\hline Spherical equivalent (D) & $<0.001$ & 1.24 & 0.21 & $0.89,1.55$ \\
\hline Axial length $(\mathrm{mm})$ & $<0.001$ & -1.42 & -0.14 & $-1.88,-0.84$ \\
\hline Anterior chamber depth ( $\mathrm{mm})$ & 0.18 & & & \\
\hline Lens thickness (mm) & 0.62 & & & \\
\hline Corneal diameter $(\mathrm{mm})$ & 0.36 & & & \\
\hline Anterior corneal curvature ( $\mathrm{mm})$ & 0.04 & -1.96 & -0.03 & $-3.12,-0.36$ \\
\hline Central corneal thickness $(\mu \mathrm{m})$ & 0.02 & -0.04 & -0.02 & $-0.05,-0.01$ \\
\hline Intraocular pressure (mmHg) & 0.004 & -0.16 & -0.02 & $-0.28,-0.09$ \\
\hline
\end{tabular}

The RNFL thickness in the multivariate analysis was found to be significantly associated with lower age $(p<$ $0.001)$, shorter AL ( $p<0.001)$, and hyperopic SE ( $p<$ 0.001). Hyperopic SE (beta $=0.13)$, shorter AL (-0.11), followed by younger age (beta $=-0.07$ ) had the highest standardised correlation coefficients, and thus had the most marked influence on RNFL thickness (Table 2).
Using the regression coefficients of the multivariate analysis, and assuming a linear age-related decline in RNFL thickness, the mean global RNFL thickness values declined by $0.11 \mu \mathrm{m}$ per year of aging. A $1-\mathrm{mm}$ axial elongation decreased the mean global RNFL thickness by approximately $1.02 \mu \mathrm{m}$. There was a $0.62 \mu \mathrm{m}$ increase in RNFL forevery dioptre change in SE towards more hyperopia (Tables 1 and 2)

Table 2: Multivariate analysis of associations between mean global retinal nerve fibre layer thickness and systemic and ocular parameters

\begin{tabular}{lclcc}
\hline Parameter & $\boldsymbol{p}$-value & $\begin{array}{l}\text { Regression } \\
\text { coefficient B }\end{array}$ & $\begin{array}{l}\text { Standardised } \\
\text { coefficient beta }\end{array}$ & 95\% CI of B \\
\hline Age (years) & $<0.001$ & -0.11 & -0.07 & $0.18,0.05$ \\
Axial length (mm) & $<0.001$ & -1.02 & -0.11 & $-1.35,-0.46$ \\
$\begin{array}{l}\text { Spherical equivalent } \\
\text { (D) }\end{array}$ & $<0.001$ & 0.62 & 0.13 & $0.51,1.32$ \\
\hline
\end{tabular}

\section{Discussion}

RNFL thickness measurements are essential to enable the early diagnosis of glaucoma, as thinning of this layer may be the earliest clinically detectable structural change in glaucoma. ${ }^{3}$ Knowledge of the normal distribution patterns of this layer is also essential to avoid confusion with physiological and pathological variations. ${ }^{13}$ To the best of our knowledge, the only published report of RNFL OCT values in South Africa was conducted by Murugan et al, ${ }^{14}$ who compared the RNFL of 40 Black and 40 Indi- an myopic students at the University of KwaZulu-Natal, South Africa. Murugan et al's ${ }^{14}$ study is however limited by consideration of a narrow age group, small sample size and refractive status (the study only considered myopic subjects in a single university sample).

The mean global RNFL thickness of $110.01 \pm 7.39$ $\mu \mathrm{m}$ found in this study is higher than the mean global RNFL thickness reported in other healthy Black, Asian and White populations. ${ }^{11,13-16}$. Racial variations in RNFL 
thickness have been reported in the literature and may be due to differences in study sample sizes, age cohorts, study designs and methods, as well as OCT device algorithms (software to detect retinal boundaries). ${ }^{11,13-17}$ However, our findings suggest that Black South Africans have thicker RNFLs than those of other Black (African-Americans, African-Caribbeans), Asian and White populations.

The mean RNFL thickness was significantly thicker in the inferior quadrant than in the superior quadrant, followed by the nasal quadrant and finally the temporal quadrant, which is consistent with the characteristic "double-hump" configuration of RNFL thickness reported in previous studies. ${ }^{14,18,19}$ This pattern reflects the nerve fibres converging to the optic nerve head $(\mathrm{ONH})$ from the superior and inferior arcuate bundles. ${ }^{18,19}$ Previous OCT and histologic studies ${ }^{11,20,21}$ have described RNFL thickness to be associated with age, with a reported average decrease of approximately $2 \mu \mathrm{m}$ for every decade of aging.

Our study showed a $1.1 \mu \mathrm{m}$ decline in mean total RNFL thickness per decade of aging. This low decline may be due to the fact that significant age-related decay of the optic nerves occurs after the age of 60 years, ${ }^{22}$ and there were only six subjects who were 60 years and older in our study. However, this association may not be uniform across the entire age spectrum and there might be some subtle associations when age is disaggregated into children and adults. The age-related decline in RNFL thickness should be taken into account when establishing normal limits, as it can be considerable in individual segments. For example, studies ${ }^{23,24}$ have shown that the largest age-dependent decline in RNFL occur in the supero-temporal and infero-temporal quadrants, in which the main blood vessels are located, and could be responsible for the shape and thickness of the RNFL profile. Bendschneider et $\mathrm{al}^{25}$ suggested that "if blood vessels make a substantial contribution to the shape and thickness of the OCT RNFL profile, further investigations subtracting blood vessels from the RNFL thickness calculation are needed to clarify if age-dependent changes in blood vessel diameter are predominantly responsible for RNFL decrease in these areas".

The relationship between RNFL thickness and AL has been examined in several studies ${ }^{18,21}$ and has yielded conflicting results. Cheung et $\mathrm{a}^{18}$ reported that longer ALs were associated with thinner mean RNFL in a population-based study of Chinese adults. Knight el $\mathrm{al}^{21}$ observed that AL had a negative correlation with the mean RNFL thickness, but a positive correlation with the temporal quadrant RNFL thickness in 63 Chinese adults. We found an association between the RNFL thickness and shorter AL, which has also been reported in previous investigations. ${ }^{18,26,27} \mathrm{AL}$ and refraction are usually correlated, which could explain the association found in this study between the average RNFL thickness and hyperopic SE. A $1-\mathrm{mm}$ axial elongation caused a mean $1.02 \mu \mathrm{m}$ RNFL thickness decrease, while a +1 dioptre increase (towards hyperopia) in refractive error caused a $0.62 \mu \mathrm{m}$ increase in RNFL thickness, as a result of which, thinning of the RNFL with greater negative refraction (and longer AL) can be expected. The RNFL thinning is possibly due to the fact that there is a stretching of the eyeball in myopia, resulting in thinner RNFL in eyes with longer $\mathrm{ALs}^{28}$

\section{Limitations}

This investigation has certain limitations. Firstly, we used non-cycloplaegic refraction which may over-estimate the myopia rate in some younger subjects. Secondly, we included a relatively low range of refractive errors and larger scale studies with a wide range of refractive errors and the inclusion of other South African ethnic groups are warranted to make comparative inferences. Thirdly, all findings and conclusions made in the study are valid for black South Africans with an age of 10 to 66 years and cannot be generalised for the entire population. This may be particularly important in the interpretation of the correlation between RNFL thickness and age. For example, the $1.1 \mu \mathrm{m}$ decline of RNFL thickness per decade of aging cannot simply be extrapolated to any age groups except the 10 to 66 year-old range.

\section{Conclusion}

The mean RNFL thickness in a healthy black South African population was $110.01 \pm 7.39 \mu \mathrm{m}$, which is higher than those reported in other races. On average, the RNFL was thicker inferiorly and superiorly, and thinner nasally and thinnest temporally. Hyperopic spherical refraction, followed by shorter AL and younger age had the most marked influence on mean global RNFL thickness. This information is important for clinical work, as it provides a point of reference for comparison with findings in diseases situations. 


\section{Conflict of interest}

None to declare.

\section{References}

1. Cook C, Foster P. Epidemiology of glaucoma: What's new? Can J Ophthalmol. 2012; 47(3): 223-226.

2. Fanihagh F, Kremmer S, Anastassiou C, Schallenberg M, Steuhl KP, Selbach M. Optical coherence tomography, scanning laser polarimetry and confocal scanning laser ophthalmoscopy in retinal nerve fiber layer measurements of glaucoma patients. Open Ophthalmol J. 2015; 9: $41-48$.

3. Nukada M, Hangai M, Mori S, Takayama K, Nakano $\mathrm{N}$, Marooka S, et al. Imaging of localized retinal nerve fiber layer defects in preperimetric glaucoma using spectral domain optical coherence tomography. J Glaucoma. 2014; 23(3): 150-159.

4. Alizadeh Y, Panjtanpanah MR, Mohammadi MJ, Behboudi H, Leili EK. Reproducibility of optical coherence tomography retinal nerve fiber layer thickness measurements before and after pupil dilation.J Ophthalmic Vis Res. 2014; 9(1): 38-43.

5. Leite MT, Rao HL, Zangwill LM, Weinreb RN, Medeiros FA. Comparison of the diagnostic accuracies of the Spectralis, Cirrus, and RTVue optical coherence tomography devices in glaucoma. Ophthalmology. 2011; 118(7): 1334-1339.

6. Thomas BJ, Galor A, Nanji AA, Sayyad FE, Wang J, Dubovy SR, et al. Ultra high-resolution anterior segment optical coherence tomography in the diagnosis and management of ocular surface squamous neoplasia. Ocular Surf. 2014; 12(1): 46-58

7. Toscano DA, De Avila MP, Chalita MRC. Reproducibility of peripapillary retinal nerve fiber layer thickness measurements using Spectral Domain OCT in Brazilian patients. Arq Bras Oftalmol. 2012; 75(5): 320-323.

8. Wang J, Abou Shousha M, Perez VL, Karp CL, Yoo $\mathrm{SH}$, Shen M, et al. Ultra high-resolution optical coherence tomography for imaging the anterior segment of the eye. Ophthalmic Surg Lasers Imaging. 2011; 42(Suppl): 15-27.

9. Savini G, Carbonelli M, Barboni P. Retinal nerve fiber layer thickness measurement by Fourier-domain optical coherence tomography: a comparison between Cirrus-HD OCT and RTVue in healthy eyes. J Glaucoma. 2010; 19(6): 369-372.

10. Arthur SN, Smith SD, Wright MM, Grajewski AL, Wang Q, Terry JM, et al. Reproducibility and agreement in evaluating retinal nerve fibre layer thickness between Stratus and Spectralis OCT. Eye (Lond). 2011; 25(2): 192200.

11. Alasil T, Wang K, Keane PA, Lee H, Baniasadi N, de Boer JF, et al. Analysis of normal retinal nerve fiber layer thickness by age, sex, and race using spectral domain optical coherence tomography. J Glaucoma. 2013; 22(7): 532-541.

12. Joubert G, Ehrlich R. Epidemiology: A research manual for South Africa, Cape Town: Oxford University Press, 2007.

13. Pinilla I, Garcia-Martin E, Idoipe M, Sancho E, Fuertes I. Comparison of retinal nerve fiber layer thickness measurements in healthy subjects using fourier and time domain optical coherence tomography. $J$ Ophthalmol. 2012, Article ID 107053, 6 pages http://dx.doi. org/10.1155/2012/107053.

14. Murugan C, Golodza BZ, Pillay K, Mthembu BN, Singh P, Maseko SK, et al. Retinal nerve fibre layer thickness of black and Indian myopic students atthe University of KwaZulu-Natal. Afr Vis Eye Health. 2015; 74(1), Art. \#24, 6 pages. http://dx.doi.org/10.4102/aveh.v74i1.24 15. O'Rese JK, Girkin CA, Budenz DL, Durbin MK, Feuer WJ, Cirrus OCT Normative Database Study Group. Effect of race, age, and axial length on optic nerve head parameters and retinal nerve fiber layer thickness measured by Cirrus HD-OCT. Arch Ophthalmol. 2012; 130(3): 312-318.

16. Rao R, Dhrami-Gavazi E, Al-Aswad L, Ciarleglio A, Cioffi GA, Blumberg DM. Optic nerve head and retinal nerve fiber layer differences between Caribbean black and African American patients as measured by spectral domain OCT. J Glaucoma. 17 Oct. 2013. Epub ahead of print.

17. Mashige KP, Oduntan OA. A review of the human retina with emphasis on the nerve fibre and macula thicknesses. Afr Vis Eye Health. 2016 ; 75(1). doi :10.4102/ aveh.v7511.330.

18. Cheung CY, Chen D, Wong TY, Tham YC, Wu R, Zheng Y, et al. Determinants of quantitative optic nerve measurements using spectral domain optical coherence tomography in a population based sample of non-glaucomatoussubjects. Invest Ophthalmol Vis Sci. 2011; 52(13): 9629-9635.

19. Leung MM, Huang RY, Lam AK. Retinal nerve fiber layer thickness in normal Hong Kong Chinese children measured with optical coherence tomography. J Glaucoma. 2010; 19(2): 95-99. 
20. Zhao L, Wang Y, Chen CX, Xu L, Jonas JB. Retinal nerve fibre layer measured by Spectralis spectral-domain optical coherence tomography: The Beijing Eye Study. Acta Ophthalmologica. 2014; 92(1): e35-e41.

21. Knight OJ, Girkin CA, Budenz DL, Durbin MK, Feuer WJ. Effect of race, age, and axial length on optic nerve head parameters and retinal nerve fiber layer thickness measured by Cirrus HDOCT. Arch Ophthalmol. 2012; 130(3): 312-318.

22. Dolman CI, McCormick AQ, Drance SM. Aging of the optic nerve. Arch Ophthalmol. 1980; 98(11): 2053-2058. 23. Hood DC, Kardon RH. A framework for comparing structural and functional measures of glaucomatous damage. Prog Retin Eye Res. 2007; 26(6): 688-710.

24. Hood DC, Fortune B, Arthur SN, Xing D, Salant JA, Ritch $\mathrm{R}$, et al. Blood vessel contributions to retinal nerve fiber layer thickness profiles measured with optical coherence tomography. J Glaucoma. 2008; 17(7): 519-528.
25. Bendschneider D, Tornow RP, Horn FK, Laemmer R, Roessler CW, Juenemann AG, et al. Retinal Nerve Fiber Layer Thickness in Normals Measured by Spectral Domain OCT. J Glaucoma. 2010; 19(7): 475-482.

26. Leung CK, Yu M, Weinreb RN, Ye C, Liu S, Lai G, et al. Retinal nerve fiber layer imaging with spectral-domain optical coherence tomography: a prospective analysis of age-related loss. Ophthalmology. 2012; 119(4): 731-737. 27. Wang YX, Pan Z, Zhao L, You QS, Xu L, Jonas JB. Retinal nerve fiber layer thickness. The Beijing Eye Study 2011. PLos One. 2013; 8(6): e66763. doi:10.1371/journal. prone.0066763.

28. Zhu BD, Li SM, Li H, Liu HR, Wang Y, Yang Z, et al. Retinal nerve fiber layer thickness in a population of 12-year-old children in central China measured by iVue100 spectral-domain optical coherence tomography: the Ayang Childhood Eye Study. Invest Ophthalmol Vis Sci. 2013; 54(13): 8104-8111. 